\title{
A fast algorithm for polynomial reconstruction of fuzzy fingerprint vault
}

\author{
Woo Yong Choi ${ }^{1}$, Sungju Lee ${ }^{2}$, Daesung Moon ${ }^{1}$, \\ Yongwha Chung ${ }^{2 \mathrm{a})}$, and Ki Young Moon ${ }^{1}$ \\ ${ }^{1}$ Biometrics Technology Research Team, ETRI, \\ 161 Gajeong-dong, Yuseong-gu, Daejeon 305-700, Korea \\ 2 Department of Computer and Information Science, Korea University, \\ Jochiwon-eup, Yeongi-gun, Chungnam 339-700, Korea \\ a)ychungy@korea.ac.kr
}

\begin{abstract}
In recent years, a cryptographic construct, called fuzzy vault, has been proposed, which aims to secure critical data (e.g., secret encryption key) with the fingerprint data in a way that only the authorized user can access the secret by providing the valid fingerprint, and some implementation results have been reported. However, all the previous results adopted the brute-force search to reconstruct the polynomial or skipped the procedure for the polynomial reconstruction. In this paper, we propose a fast polynomial reconstruction algorithm for the fuzzy fingerprint vault which can improve the execution time of the brute-force search by a factor of 300 1,500.
\end{abstract}

Keywords: crypto-biometrics, fuzzy vault, polynomial reconstruction

Classification: Science and engineering for electronics

\section{References}

[1] A. Juels and M. Sudan, "A fuzzy vault scheme," Proc. IEEE Int. Symp. Info. Theory, p. 408, 2002.

[2] Y. Chung, D. Moon, S. Lee, S. Jung, T. Kim, and D. Ahn, "Automatic alignment of fingerprint features for fuzzy fingerprint vault," Proc. $1^{\text {st }}$ Int. Conf. Info. Security and Cryptology, pp. 358-369, 2005.

[3] U. Uludag, S. Pankanti, and A. Jain, "Fuzzy vault for fingerprints," Proc. $5^{\text {th }}$ Int. Conf. AVBPA, pp. 310-319, 2005.

[4] A. Kanak and I. Sogukpinar, "Fingerprint hardening with randomly selected chaff minutiae," Proc. 12 ${ }^{\text {th }}$ Int. Conf. CAIP, pp. 383-390, 2007.

[5] K. Nandakumar, A. Jain, and S. Pankanti, "Fingerprint-based fuzzy vault," IEEE Trans. Inf. Forensics Security, vol. 2, no. 4, pp. 744-757, 2007.

[6] Q. Li, Z. Liu, and X. Niu, "Analysis and problems on fuzzy vault scheme," Proc. $2^{\text {nd }}$ Int. Conf. IIH-MSP, pp. 244-250, 2006.

[7] D. Maio, D. Maltoni, J. Wayman, and A. Jain, "FVC2002: second fingerprint verification competition," Proc. Int. Conf. Pattern Rec., pp. 811814, 2002. 


\section{Introduction}

Recently, Juels and Sudan [1] proposed the fuzzy vault scheme. In the fuzzy vault scheme, Alice can place a secret value in a vault and lock it using an unordered set $A$. She selects a polynomial such that the secret value is embedded in the polynomial coefficients. Then, Bob, using an unordered set $B$, can unlock the vault (i.e., reconstruct the polynomial) only if $B$ overlaps with $A$ to a great extent.

Based on the fuzzy vault, some implementation results for fingerprint have been reported $[2,3,4,5]$. However, all the previous results adopted the brute-force search [1] to reconstruct the polynomial or skipped the procedure for polynomial reconstruction because of its difficulty $[3,6]$. In this paper, we propose a fast polynomial reconstruction algorithm for the fuzzy fingerprint vault. Although our algorithm needs one more real point than the brute-force search, the polynomial can be reconstructed in real time.

\section{Fuzzy fingerprint vault}

Fig. 1 shows the block diagram of the fuzzy fingerprint vault system we considered. The details of each procedure are as follows.

\subsection{Enrollment procedure}

Given the fingerprint image to be enrolled, we first extract minutiae from the image to form a locking set of the form:

$$
L=\left\{m_{i} \mid 1 \leq i \leq n\right\},
$$

where $m_{i}=\left(x_{i}, y_{i}, \theta_{i}, t_{i}\right)$ is the $i^{\text {th }}$ enrollment minutia, and $n$ is the number of the enrollment minutiae. Then, a number of chaff minutiae are generated and constitute a minutia set along with the real minutiae. After adding the chaff minutiae, the total number of minutiae is $r$. In this paper, all

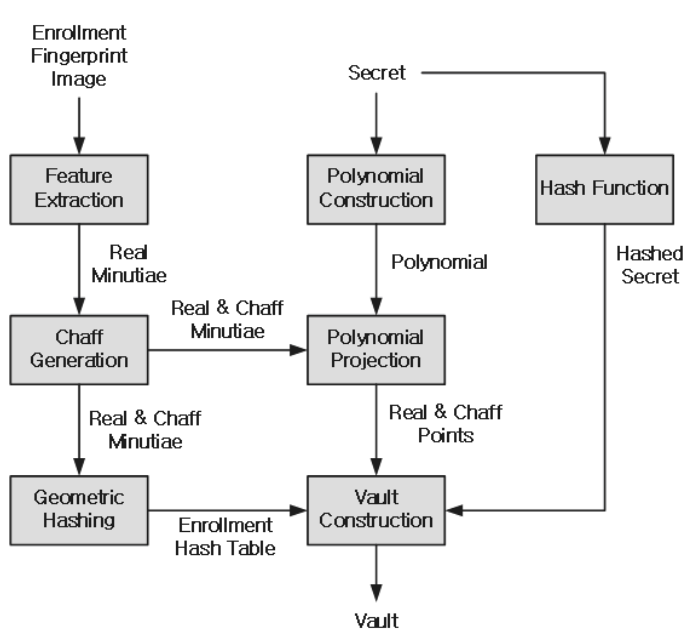

(a) Enrollment procedure

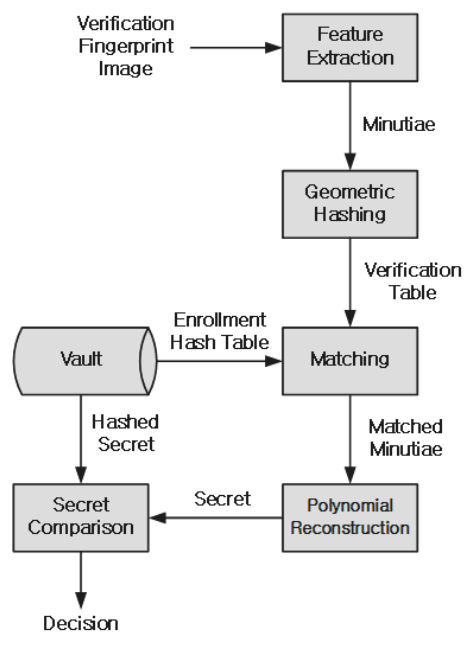

(b) Verification procedure

Fig. 1. Block diagrams of the fuzzy fingerprint vault system 
arithmetic operations are conducted in a finite field of order $2^{18}$, namely $\mathrm{GF}\left(2^{18}\right)$. Thus, each coordinate is scaled to the range $[0,512]$ for the purpose of the arithmetic in $\mathrm{GF}\left(2^{18}\right)$. From the minutiae set with $r$ minutiae, we generate the enrollment hash table [2]. Then, we randomly generate a secret $\kappa$ of length $18 k$ bits, and then it is encoded into a polynomial $p$ of degree $(k-1)$ over $\mathrm{GF}\left(2^{18}\right)$ :

$$
p(u)=a_{0}+a_{1} u+a_{2} u^{2}+\cdots+a_{k-1} u^{k-1} .
$$

This polynomial becomes the secret to be protected. As in [3], we concatenate $x$ and $y$ coordinates of a minutia to arrive at the 18-bit locking/unlocking data unit $u$. Then, we project the real and chaff points (i.e., minutiae) on and off the polynomial, respectively. That is,

$$
v_{i}= \begin{cases}p\left(u_{i}\right), & \text { if } u_{i} \text { is real } \\ p\left(u_{i}\right)+\alpha_{i}, & \text { if } u_{i} \text { is chaff, }\end{cases}
$$

where $\alpha_{i}$ is a non-zero element of $\operatorname{GF}\left(2^{18}\right)$. Finally, the vault is constituted by the real and chaff points, the enrollment hash table, and the secret. The secret should be stored in a hashed form, instead of in the clear form.

\subsection{Verification procedure}

Given the fingerprint image to be verified, we first extract minutiae from the image, and a verification table [2] is generated according to the geometric characteristic of the minutiae. Then, the verification table is compared with the enrollment hash table, and an unlocking set $U$ is finally selected.

$$
U=\left\{m_{i}^{\prime} \mid 1 \leq i \leq t\right\},
$$

where $m_{i}^{\prime}$ is the $i^{\text {th }}$ matched minutia, and $t$ is the number of the matched minutiae. The vault can be successfully unlocked only if $U$ overlaps with $L$ to a great extent.

Then, we take $t$ points corresponding to the unlocking set $U$ from the vault. These $t$ points may contain some chaff points as well as the real points even if the user is genuine. Hence, in order to reconstruct the polynomial, we have to select $k$ real points from among the $t$ points. After the polynomial is reconstructed, it is compared with the true polynomial stored in the vault. A decision to accept/reject the user depends on the result of this comparison. If $|U \cap L| \geq k$, the (k-1)-degree polynomial can be successfully reconstructed by using the brute-force search. However, it becomes impracticable as $t$ and/or $k$ increase. Uludag [3] used only 18 minutiae to prevent $t$ from being too large.

\section{Proposed method of polynomial reconstruction}

The following theorem provides the conditions under which a linear system of $m$ equations in $n$ unknowns is guaranteed to be consistent.

Consistency Theorem. If $\boldsymbol{A} \boldsymbol{x}=\boldsymbol{b}$ is a linear system of $m$ equations in $n$ unknowns, then the following are equivalent. 
(a) $\boldsymbol{A} \boldsymbol{x}=\boldsymbol{b}$ is consistent.

(b) $\boldsymbol{b}$ is in the column space of $\boldsymbol{A}$.

(c) The coefficient matrix $\boldsymbol{A}$ and the augmented matrix $[\boldsymbol{A} \mid \boldsymbol{b}]$ have the same rank.

To begin with, let us consider a linear system of $(k+1)$ equations in $k$ unknowns:

$$
\mathbf{U} \cdot \mathbf{a}=\mathbf{v}
$$

where

$$
\mathbf{U}=\left[\begin{array}{ccccc}
1 & u_{1} & u_{1}^{2} & \cdots & u_{1}^{k-1} \\
1 & u_{2} & u_{2}^{2} & \cdots & u_{2}^{k-1} \\
\vdots & \vdots & \vdots & \ddots & \vdots \\
1 & u_{k+1} & u_{k+1}^{2} & \cdots & u_{k+1}^{k-1}
\end{array}\right], \mathbf{a}=\left[\begin{array}{c}
a_{0} \\
a_{1} \\
\vdots \\
a_{k-1}
\end{array}\right], \mathbf{v}=\left[\begin{array}{c}
v_{1} \\
v_{2} \\
\vdots \\
v_{k+1}
\end{array}\right]
$$

Then, the corresponding augmented matrix $\mathbf{W}$ is of the form:

$$
\mathbf{W}=\left[\begin{array}{ccccc:c}
1 & u_{1} & u_{1}^{2} & \cdots & u_{1}^{k-1} & v_{1} \\
\vdots & \vdots & \vdots & \ddots & \vdots & \vdots \\
1 & u_{k+1} & u_{k+1}^{2} & \cdots & u_{k+1}^{k-1} & v_{k+1}
\end{array}\right]
$$

It is straightforward that the rank of the matrix $\mathbf{U}$ is $k$. According to the Consistency Theorem, the rank of the augmented matrix $\mathbf{W}$ must be equal to $k$ to guarantee that the linear system has a solution. The Gaussian elimination was used to check whether the augmented matrix had rank $k$ or not. The elementary row operations, provided we do not perform the operation of interchanging two rows, were used to reduce the augmented matrix $\mathbf{W}$ into the row-echelon form:

$$
\left[\begin{array}{ccccc:c}
1 & u_{1} & u_{1}^{2} & \cdots & u_{1}^{k-1} & v_{1} \\
0 & 1 & u_{2}^{2(2)} & \cdots & u_{2}^{k-1(2)} & v_{2}^{(2)} \\
\vdots & \vdots & \vdots & & \vdots & \vdots \\
0 & 0 & 0 & \cdots & 1 & v_{k}^{(k)} \\
0 & 0 & 0 & \cdots & 0 & v_{k+1}^{(k+1)}
\end{array}\right]
$$

where $u_{j}^{l(i)}$ and $v_{j}^{(i)}$ are the values of $u_{j}^{l}$ and $v_{j}$ when $j^{\text {th }}$ row has "leading 1 " at $i^{\text {th }}$ element, respectively. Note that the diagonal elements of Eq. (8) cannot be zero because the rank of the matrix $\mathbf{U}$ is $k$. From the parts (a) and (c) of the Consistency Theorem, it follows that if $v_{k+1}^{(k+1)} \neq 0$, the linear system of Eq. (5) does not have a solution. Hence, there exists at least one chaff point in the set $\left\{\left(u_{i}, v_{i}\right) \mid i=1, \ldots, k+1\right\}$, and we need not perform the polynomial reconstruction process. On the contrary, if $v_{k+1}^{(k+1)}=0$, then all the points are probably the real points. Thus, we try to reconstruct the polynomial with $k$ points and compare it with the true polynomial.

Up to this point, we have explained how to reconstruct a $(k-1)$-degree polynomial from $(k+1)$ matched minutiae. In general, the unlocking set has $t$ minutiae, so let us consider a linear system of $t$ equations in $k$ unknowns:

$$
\left[\begin{array}{ccccc}
1 & u_{1} & u_{1}^{2} & \cdots & u_{1}^{k-1} \\
\vdots & \vdots & \vdots & \ddots & \vdots \\
1 & u_{t} & u_{t}^{2} & \cdots & u_{t}^{k-1}
\end{array}\right]\left[\begin{array}{c}
a_{0} \\
\vdots \\
a_{k-1}
\end{array}\right]\left[\begin{array}{c}
v_{1} \\
\vdots \\
v_{t}
\end{array}\right]
$$


where $t>k$. Clearly, if $t<k$, then the polynomial cannot be reconstructed. Also, if $t=k$, we can reconstruct the polynomial with the $k$ points. Suppose that we select $(k+1)$ real points from among $t$ points, we can reconstruct the true polynomial. However, if at least one chaff point exists in the $(k+1)$ selected points, the true polynomial cannot be reconstructed. The procedure for the proposed polynomial reconstruction algorithm is as follows.

1 ) We select $k$ points from among $t$ points with real and chaff points mixed, and place them to the top of Eq. (9).

2) The augmented matrix of Eq. (9) are obtained, and reduced into the following row-echelon form:

$$
\left[\begin{array}{ccccc:c}
1 & u_{1} & u_{1}^{2} & \cdots & u_{1}^{k-1} & v_{1} \\
0 & 1 & u_{2}^{2(2)} & \cdots & u_{2}^{k-1(2)} & v_{2}^{(2)} \\
\vdots & \vdots & \vdots & & \vdots & \vdots \\
0 & 0 & 0 & \cdots & 1 & v_{k}^{(k)} \\
0 & 0 & 0 & \cdots & 0 & v_{k+1}^{(k+1)} \\
\vdots & \vdots & \vdots & & \vdots & \vdots \\
0 & 0 & 0 & \cdots & 0 & v_{t}^{(k+1)}
\end{array}\right]
$$

3) To determine whether the $k$ points $\left(u_{1}, v_{1}\right), \ldots,\left(u_{k}, v_{k}\right)$ are valid candidates or not, we will check the values of $v_{k+1}^{(k+1)}, \cdots, v_{t}^{(k+1)}$. Therefore, the proposed algorithm needs one more real point than the bruteforce search to reconstruct the polynomial. If there is at least one zero among $v_{k+1}^{(k+1)}, \cdots, v_{t}^{(k+1)}$, we reconstruct the polynomial with the selected $k$ points, and compare it with the true polynomial.

4) Steps 1) 3) are repeated until the true polynomial is reconstructed.

However, the computations of step 2) take too much time to be implemented in real time. Fortunately, in order to obtain the values of $v_{k+1}^{(k+1)}, \cdots$, $v_{t}^{(k+1)}$, we do not have to apply the Gaussian elimination. We have found the following recursive formula, so the computation time can be considerably reduced:

$$
v_{j}^{(i+1)}= \begin{cases}v_{j}, & i=0 \\ \frac{v_{j}^{(i)}-v_{i}^{(i)}}{u_{j}-u_{i}}, & i=1, \cdots, \min (k, j-1) .\end{cases}
$$

Eq. (11) gives exactly the same solution as the Gaussian elimination. The proof is omitted due to space limitations.

\section{Experimental results}

To evaluate the performance of the proposed polynomial reconstruction algorithm, we used FVC2002-DB1 fingerprint database [7]. The database consisted of 100 fingers, and 8 impressions per finger. The resolution of the sensor was $500 \mathrm{dpi}$, and the size of captured fingerprint images was $388 \times 374$. Each sample was matched against the remaining samples of the same finger to compute the False Rejection Rate (FRR). Similarly, the first sample of each finger was matched against the first sample of the remaining fingers to 
compute the False Acceptance Rate (FAR). The number of genuine tests was 2,800 , whereas the number of impostor tests was 4,950. All experiments were performed on a system with a $2.66 \mathrm{GHz}$ processor.

As in [2], we selected the number of the chaff minutiae as 200. The average number of minutiae extracted by our feature extractor is about 30, so the average number of points in the vault is about 230 . The number of the matched minutiae including the real and the chaff minutiae are listed in Table I.

To examine the effectiveness of the proposed algorithm, we compared it with the brute-force search. Table II shows the error rates of our fuzzy fingerprint vault system. Recall that the proposed algorithm needs one more real minutia than the brute-force search. Hence, we can confirm that the error rates of the proposed algorithm using the $k$-degree polynomial is equal to those of the brute-force search using the $(k+1)$-degree polynomial.

Table III shows the average execution time for polynomial reconstruction. Although Lagrange interpolation is an efficient technique to evaluate polynomial, brute-force search spends too much time, because a number of evaluations are needed to reconstruct a polynomial. In fact, the Lagrange

Table I. Average number of minutiae. Numbers in the parenthesis represent the minimum and maximum values

\begin{tabular}{c|c|c}
\hline \hline & Genuine & Impostor \\
\hline \# of enrollment minutiae $(r)$ & $230(206,260)$ & $230(214,253)$ \\
\hline \# of matched minutiae $(t)$ & $18.3(2,51)$ & $9.5(5,18)$ \\
\hline \# of real minutiae & $17.1(0,48)$ & $3.2(0,13)$ \\
\hline \# of chaff minutiae & $1.2(0,11)$ & $6.3(0,14)$ \\
\hline \hline
\end{tabular}

Table II. Error rates of the fuzzy fingerprint vault system (unit: \%)

\begin{tabular}{c|c|c|c|c}
\hline \hline \multirow{2}{*}{$\begin{array}{c}\text { Polynomial } \\
\text { degree }\end{array}$} & \multicolumn{2}{|c|}{ Brute-force search } & \multicolumn{2}{c}{ Proposed algorithm } \\
\cline { 2 - 5 } & FRR & FAR & FRR & FAR \\
\hline 7 & 10.0 & 5.6 & 12.7 & 2.7 \\
\hline 8 & 12.7 & 2.7 & 15.5 & 1.4 \\
\hline 9 & 15.5 & 1.4 & 19.1 & 0.6 \\
\hline \hline
\end{tabular}

Table III. Average execution time for polynomial reconstruction (unit: second)

\begin{tabular}{c|c|c|c|c}
\hline \hline \multirow{2}{*}{$\begin{array}{c}\text { Polynomial } \\
\text { degree }\end{array}$} & \multicolumn{2}{|c|}{ Brute-force search } & \multicolumn{2}{c}{ Proposed algorithm } \\
\cline { 2 - 5 } & Genuine & Impostor & Genuine & Impostor \\
\hline 7 & 4.7 & 0.031 & 0.015 & 0.001 \\
\hline 8 & 44.6 & 0.051 & 0.064 & 0.001 \\
\hline 9 & 390.9 & 0.077 & 0.256 & 0.001 \\
\hline \hline
\end{tabular}


interpolation time for evaluating a 9-degree polynomial was 0.0012 seconds, but 321,291 evaluations were performed, on average, to reconstruct a polynomial. From this table, we can see that the genuine test needs more time to reconstruct the polynomial than the impostor test because the former has more matched minutiae than the latter. Furthermore, as the polynomial degree gets higher, the proposed algorithm can improve the performance of the brute-force search significantly.

\section{Conclusion}

In this paper, we proposed a new polynomial reconstruction algorithm for the fuzzy fingerprint vault. To reduce the execution time, it determines the candidate sets with chaff points effectively using the recursive formula and excludes them from the reconstruction trial. The experimental results showed that the proposed algorithm enhanced the execution time of the brute-force search by a factor of $300 \sim 1,500$ though it needed one more real point than the brute-force search.

\section{Acknowledgments}

This work was supported by the IT R\&D program of MKE/IITA [2007-S-02001 Development of privacy enhanced biometric system] and the Chung-Ang University HNRC-ITRC. 